\title{
Особенности применения кварцевых генераторов с высокой температурной стабильностью
}

\author{
А. Котюков ${ }^{1}$, А. Никонов², А. Заславский ${ }^{3}$, Ю. Иванов ${ }^{4}$
}

УДК 621.373 | ВАК 05.27 .01

\begin{abstract}
Температурная стабильность частоты - один из ключевых параметров кварцевых генераторов. Современные термостатированные кварцевые генераторы могут обеспечивать очень высокую температурную стабильность частоты вплоть до $\pm 1 \cdot 10^{-11}$ в широком интервале температур от -40 до $85{ }^{\circ} \mathrm{C}$. Столь малые величины изменения частоты требуют некоторых особенностей при измерении и соответственно при эксплуатации таких генераторов.

В статье рассматриваются особенности эксплуатации подобных генераторов, методики измерения температурной стабильности, а также влияние других факторов на нее.
\end{abstract}

дним из основных параметров кварцевых генераторов является температурная стабильность. Существует несколько методов ее обеспечения:

1. Простые генераторы (Кг). Температурная стабильность обеспечивается только самим кварцевым резонатором за счет выбора угла среза кварцевого элемента. Температурная стабильность для таких генераторов может составлять $\pm(10 \ldots 15) \cdot 10^{-6}$ в интервале температур от -40 до $85^{\circ} \mathrm{C}$.

2. Термокомпенсированные кварцевые генераторы (ТККг). В таких генераторах присутствуют дополнительные элементы, которые формируют управляющее напряжение, компенсирующее зависимость выходной частоты от температуры. Температурная стабильность для таких генераторов может составлять $\pm(1 \ldots 3) \cdot 10^{-7}$ в интервале температур от -40 до $85^{\circ} \mathrm{C}$.

3. Термостатированные кварцевые генераторы (ТСКг). В таких генераторах кварцевый резонатор и основная часть элементов находятся в термостате, поддерживающем постоянную температуру. Температурная стабильность для таких генераторов может составлять до $\pm(1 . .5) \cdot 10^{-11}$ в интервале температур от -40 до $85^{\circ} \mathrm{C}$

Далее мы рассмотрим именно термостатированные генераторы, причем только самые высокостабильные.

Рассмотрим более детально базовую конструкцию термостатированных генераторов. Как уже было сказано,

AO «Морион», начальник лаборатории разработки генераторов. AO «Морион», заместитель начальника лаборатории разработки генераторов

АО «Морион», ведущий инженер-разработчик КГ.

АО «Морион», старший инженер-разработчик КГ. в подобных генераторах все чувствительные к изменению температуры элементы находятся внутри термостата, в котором поддерживается постоянная температура (рис. 2).

Температура внутри термостата устанавливается несколько выше (обычно на $5-15^{\circ} \mathrm{C}$ ) верхней рабочей
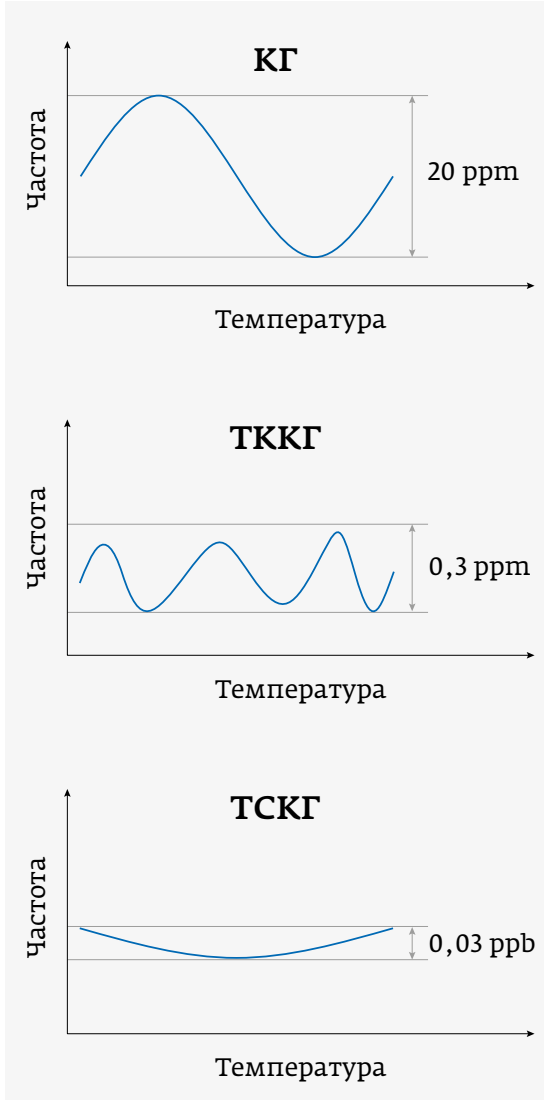

Рис. 1.

Зависимость частоты от температуры для разных видов кварцевых генераторов 


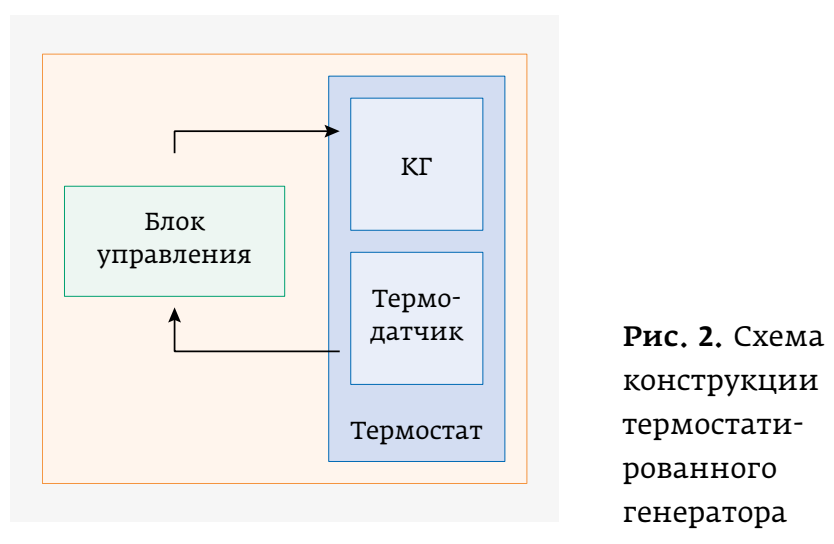

температуры эксплуатации генератора. Кроме того, она настраивается таким образом, чтобы температурная зависимость резонатора находилась в районе какого-то из его экстремумов (точки LTP и UTP на рис. 3). Таким образом обеспечивается минимальное изменение частоты кварцевого генератора от изменения температуры окружающей среды.

Необходимость поддержания высокой температуры термостатом приводит к следующим отличительным чертам термостатированных кварцевых генераторов:

- повышенный ток потребления при включении (как только температура внутри термостата достигает заданного уровня, ток потребления существенно уменьшается);

- необходимость первоначального прогрева (характеризуется точностью установления частоты в определенных пределах за фиксированное время. Обычно составляет от 2 до 5 мин при температуре $25^{\circ} \mathrm{C}$ с точностью до $\pm 2 \cdot 10^{-8}$ ).

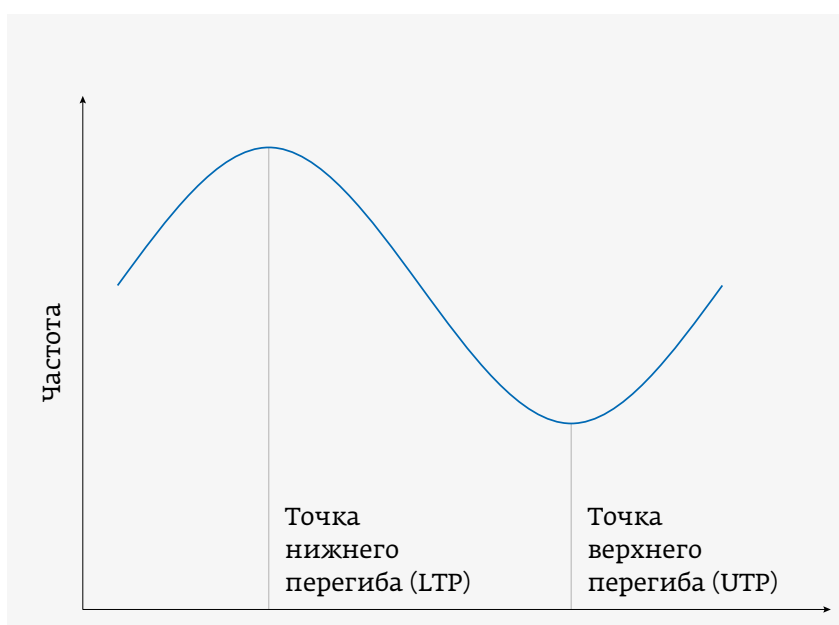

Температура

Рис. 3. Типовая зависимость частоты от температуры для кварцевого резонатора
Подобная базовая модель может обеспечить температурную стабильность от $\pm 1 \cdot 10^{-8}$ до $\pm 5 \cdot 10^{-10}$, в зависимости от конструкции. Существует несколько путей повышения температурной стабильности описанной конструкции:

1. Использование двойного термостата (DOCXO). В подобных генераторах используется дополнительный термостат, внутрь которого помещается базовый термостат. Это достаточно эффективный путь, который обычно позволяет достичь стабильности до $\pm 7 \cdot 10^{-10}$. Однако он несет в себе неизбежные издержки - относительно большие габариты и ограничение верхней рабочей температуры эксплуатации генератора из-за необходимости делать большую разницу между рабочей температурой и температурой термостата.

2. Использование дополнительной температурной компенсации. Итоговая зависимость частоты от температуры базовой конструкции обычно носит более или менее линейный характер, что дополнительно позволяет относительно просто ее компенсировать. Из недостатков этого метода следует отметить довольно высокую крутизну итоговой зависимости частоты от температуры, что может нивелировать все улучшения. Применение подобного метода к термостатированным генераторам обычно позволяет увеличить температурную стабильность до пяти раз.

3. Наиболее сложный, но дающий наилучшие результаты метод "вылизывания" базовой конструкции. Он заключается в тщательном расчете и долгом многоитеративном процессе доработки конструкции конкретного типа генераторов для получения лучшей температурной стабильности. В частности, за счет понижения температурных градиентов. Благодаря этому можно достичь температурной стабильности, получаемой у генераторов с двойным термостатированием, но при этом сохранив габариты и, в особенности, высоту базовой конструкции.

Для получения эксклюзивно высокой температурной стабильности, например до 1·10-11, приходится в полной мере реализовывать все описанные выше меры улучшения температурной стабильности.

Как при эксплуатации, так и при измерении параметров генераторов с высокой температурной стабильностью могут возникать дополнительные факторы, влияющие на температурную стабильность, которые необходимо учитывать. Так, например, выходная частота кварцевых генераторов изменяется с течением времени. При этом крайне важно, сколько времени генератор находился во включенном состоянии. Так, для генераторов, включенных в течение нескольких недель, суточное изменение частоты будет составлять 


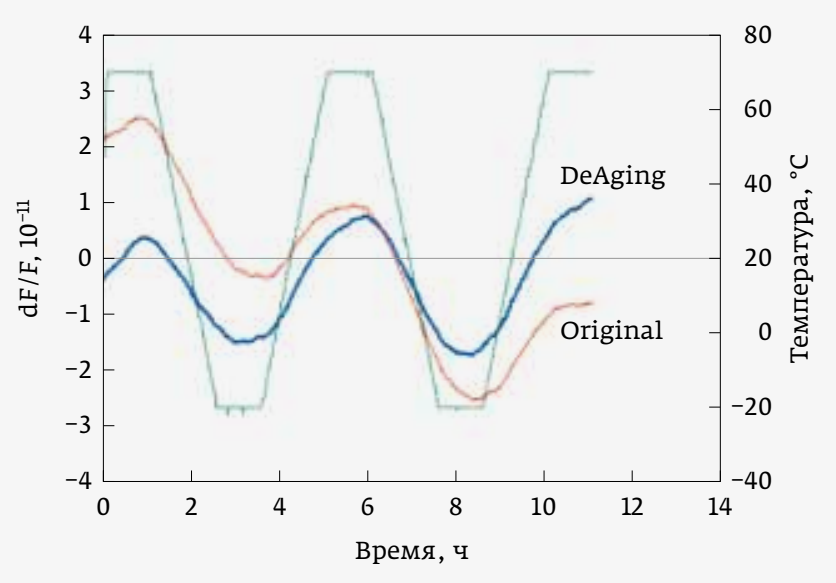

Рис. 4. Результат измерения температурной стабильности генератора ГК360-ТС производства АО "Морион"

несколько единиц 10-11, а для генераторов, включенных в течение только одного дня, - эта величина уже будет несколько единиц 10-10. Нетрудно заметить, что такой вклад будет заметен при измерении температурной стабильности, тем более, когда она мала и сопоставима с таким уходом. Таким образом, при оценке стоит учитывать дрейф частоты генератора. Сделать это довольно просто - необходимо выдержать генератор при некоторой постоянной температуре и фиксировать его частоту. Далее по полученным результатам можно построить модель ухода частоты с течением времени - для недолгих промежутков времени вполне хватит простой линейной модели. Обычно при испытаниях генераторов с очень высокой температурной стабильностью нам приходится делать несколько циклов нагрева / охлаждения, чтобы убедиться, что генератор удовлетворяет нормам по температурной стабильности. Влияние дрейфа частоты отчетливо видно на результатах испытаний генератора ГК360-ТС производства «Морион».

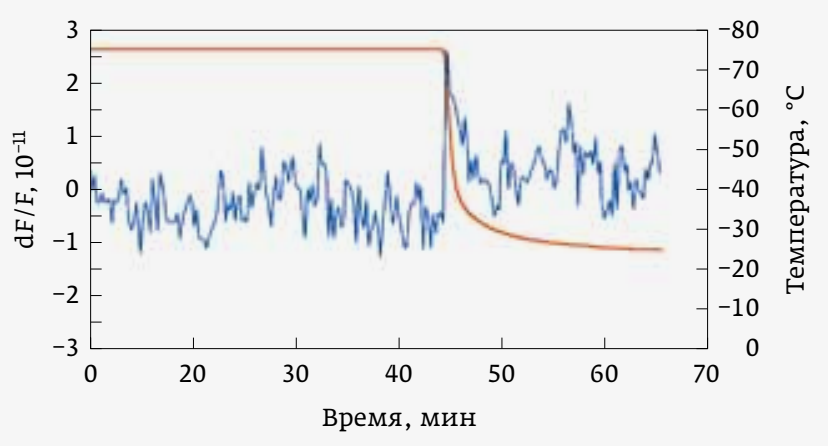

Рис. 6. Пример малой реакции на температурный удар для ГК $360-\mathrm{TC}$

На рис. 4. красным цветом показана исходная характеристика, снятая в процессе измерений, ее линейный наклон связан со старением генератора, синим цветом - эта же характеристика, но с вычтенным дрейфом.

Как уже было отмечено, если для повышения температурной стабильности используется дополнительная компенсация, то на итоговой характеристике могут присутствовать отдельные участки с сильной крутизной. Подобная ситуация не сильно выражена для термостатированных кварцевых генераторов, но очень заметна в случае с рубидиевыми генераторами.

На рис. 5 показаны две разные зависимости частоты от температуры. В первом случае (рис. 5а), при малом изменении температуры, изменение частоты будет также пропорционально мало. Во втором случае (рис. 5б), при малом изменении температуры, частота может измениться существенно больше, вплоть до значения температурной стабильности во всем интервале.

Также из-за компенсации или неудачной конструкции можно наблюдать большие изменения частоты, многократно превышающие норму при быстром изменении температуры (рис. 6).

У термостатированных генераторов с высокой температурной стабильностью наблюдается изменение формы и величины зависимости частоты от температуры при изменении их ориентации. Происходит это вследствие конвекции внутри объема генератора. У правильно разработанного генератора такая зависимость должна быть сведена к минимуму и учтена при испытаниях.

Говоря о термостатированных генераторах сверхвысокой 


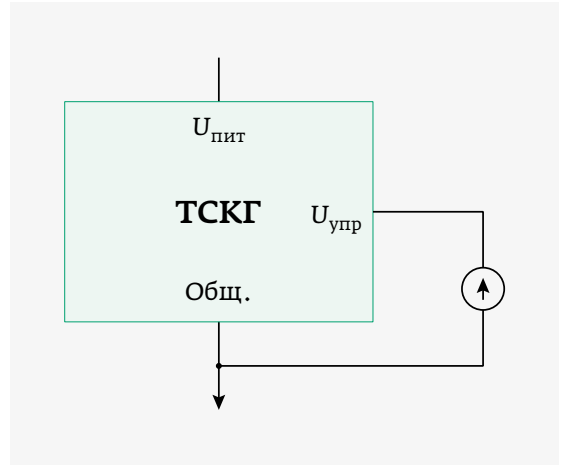

Рис. 7. Схема
включения
генератора
с аналоговым
управлением
с общей
«землей"

стабильности, отдельно стоит сказать о проблемах, возникающих при подстройке частоты генератора с помощью управляющего напряжения. Наличие этой функции напрямую влияет на стабильность генератора. Когда мы говорим о столь малых величинах нестабильности, вклад наличия перестройки становится особенно заметным. Так, генератор без такой опции будет обладать большей температурной и кратковременной стабильностью, чем с ней. Например, температурную стабильность генератора без перестройки можно довести до $\pm 7 \cdot 10^{-11}$, тогда как с перестройкой эта величина уже будет равна $\pm 2 \cdot 10^{-11}$. Соответственно, при необходимости лучшей температурной стабильности предпочтение должно быть отдано системам, в которых допустимо применение генератора без возможности перестройки частоты.

Перестройка может обеспечиваться либо аналоговой цепью, либо цифровой. Генераторы с цифровым управлением содержат ЦАП, и управление частотой осуществляется подачей кода. Управление ЦАП возможно по протоколу I² или SPI. При цифровом варианте управления деградация температурной стабильности минимальна, однако при изменении кода управления возможно ухудшение кратковременной нестабильности и фазовых шумов.

Еще одним ограничением является минимальный фиксированный шаг перестройки, который зависит от разрядности ЦАП и для 20-разрядного ЦАП составляет от $5 \cdot 10^{-13}$ до $10 \cdot 10^{-13}$.

В генераторах с аналоговым управлением для приведения частоты генератора к номиналу на вход управления необходимо подать управляющее напряжение (рис. 7).

На земляном выводе генератора возникает падение напряжения, зависящее от протекающего тока нагревательных транзисторов термостата, и при таком подключении оно попадает в цепь управления и ухудшает температурную и кратковременную стабильность частоты.

Для уменьшения этого влияния необходимо уменьшить сопротивление (длину) общей цепи протекающих токов питания генератора и цепи управления. Кардинальным способом является использование раздельных

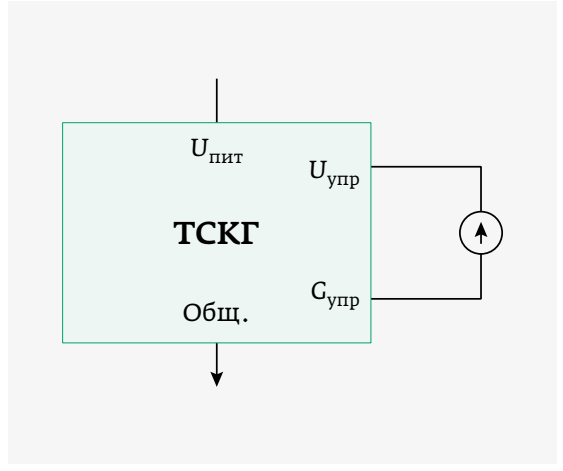

\section{Рис. 8. Схема включения генератора с аналоговым управлением с раздельными выводами «земля"}

выводов «земля» (рис. 8). Но такой путь снижает унификацию генераторов и накладывает достаточно серьезные ограничения и схемотехнику оборудования.

Еще одним нюансом эксплуатации сверхвысокостабильных генераторов являются применяемые материалы, так как при соединении разнородных проводников, спаи которых находятся при разных температурах, в цепи управления возникает термоЭдс, которая будет влиять на температурную стабильность частоты.

Чем выше температурная стабильность, тем сильнее будут влиять все вышеописанные явления.

Генераторы с высокой температурной стабильностью могут успешно применяться во многих областях, где необходима очень стабильная частота. Они даже могут посоревноваться с рубидиевыми генераторами в некоторых областях применения, обладая меньшими габаритами и потреблением. Их зависимость частоты от изменения температуры гораздо более линейна и обладает меньшей крутизной. Таким образом, при небольших изменениях температуры окружающей среды она будет существенно выше, чем у рубидиевых генераторов. Единственное, что можно отметить, это то, что, в отличие от рубидиевых, термостатированные кварцевые генераторы обладают большим старением, но в случае крайне малого изменения частоты при изменении температуры его влияние можно компенсировать. В статье были рассмотрены особенности конструкции термостатированных генераторов и методы улучшения температурной стабильности. А также указаны некоторые особенности использования подобных генераторов.

\section{ЛИТЕРАТУРА}

1. John R. Vig. Quartz crystal resonators and oscillators: a tutorial, US Army Communications-Electronics Research // Development \& Engineering Center Fort Monmouth. NJ. USA. March 2004.

2. Kotyukov A., Ivanov Y., Nikonov A. Precise Frequency Sources Meeting the $5 \mathrm{G}$ Holdover Time Interval Error Requirement // Microwave journal. May 2018. V. 61. PP. 128-138. 